\title{
Differential expression of human chorionic gonadotropin (hCG) glycosylation isoforms in failing and continuing pregnancies: preliminary characterization of the hyperglycosylated hCG epitope
}

\author{
G Kovalevskaya ${ }^{1}$, S Birken², T Kakuma ${ }^{3}$, N Ozaki², M Sauer², \\ S Lindheim ${ }^{2}$, M Cohen $^{2}$, A Kelly ${ }^{2}$, J Schlatterer ${ }^{1}$ \\ and J F O'Connor ${ }^{1,4}$ \\ ${ }^{1}$ Irving Center for Clinical Research, Columbia University College of Physicians and Surgeons, New York, New York 10032, USA \\ ${ }^{2}$ Department of Obstetrics and Gynecology, Columbia University College of Physicians and Surgeons, New York, New York 10032, USA \\ ${ }^{3}$ New York Hospital - Cornell Medical Center, White Plains, New York 10605, USA \\ ${ }^{4}$ Department of Pathology, Columbia University College of Physicians and Surgeons, New York, New York 10032, USA \\ (Requests for offprints should be addressed to J F O'Connor, Irving Center for Clinical Research, Columbia University College of Physicians and Surgeons, \\ 630 W 168th St, PH10-305, New York, New York 10032, USA; Email: jfo1@columbia.edu)
}

\begin{abstract}
Human chorionic gonadotropin (hCG) glycoforms change as pregnancy progresses. We have developed an antibody (B152) which can measure a hyperglycosylated early pregnancy isoform of hCG. This putative hyperglycosylated form of hCG arises very early in pregnancies and is rapidly replaced by an isoform that predominates for the remainder of the pregnancy. The profiles of these hCG glycoforms are measured as a ratio of values of two immunometric assays. The profiles of these ratios differ between pregnancies which persist and those which will experience early failure.

In this report, daily urine hCG isoform ratios from donor eggs (no exogenous hCG pretreatment), in vitro fertilization pregnancies were profiled and analyzed from the first day following embryo transfer (ET). Significant differences were found between continuing pregnancy and pregnancy loss throughout days $5-20$ post-ET. When hCG isoform ratios were analyzed from the first day of detectable hCG, pregnancy loss could be predicted in the case of a single fetus both during the 5- to 10-day time segment $(P=0.018)$ and the 10 - to 15 -day time segment $(P=0 \cdot 045)$. When single and multiple fetus pregnancies were analyzed together significance was approached in the 10 - to 15 -day time period $(P=0 \cdot 058)$.
\end{abstract}

In a second population of pregnant women who conceived naturally, in whom urine samples were collected at approximately weekly intervals to either term birth or clinical spontaneous abortion, the ratio could discriminate between miscarriages and normal term pregnancies $(P=0 \cdot 043)$. In later pregnancy, the ratio of hCG isoforms declined more rapidly in miscarriages than in term pregnancy.

Antibody B152 was produced using a choriocarcinomaderived hCG (C5), which was hyperglycosylated at both $\mathrm{N}$ - and O-linked sites and was 100\% nicked at position $\beta_{47-48}$. Western blot analyses supported the assay results showing that early pregnancy urine does not contain nicked C5-like hCG. Also, the early pregnancy hCG appeared to be the same size as later pregnancy hCG as judged by SDS gel electrophoresis. A series of Western blot analyses and immunoassays conducted with the samples either non-reduced or reduced showed that B152 is directed to a linear epitope located in the $\mathrm{COOH}-$ terminal peptide region of the $\beta$ subunit. This indicated that only the $\mathrm{O}$-glycan groups and not the $\mathrm{N}$-linked glycans are part of the antibody epitope.

Journal of Endocrinology (2002) 172, 497-506

\section{Introduction}

We have previously documented that the expression of various urinary human chorionic gonadotropin (hCG) molecular forms differs between naturally conceived pregnancies, which carry to term, and those destined for early pregnancy loss (Ellish et al. 1996, O'Connor et al. 1998). These differences encompass both the relative production of hCG metabolic fragments and the expression of hCG hyperglycosylated isoforms. We now report preliminary studies which indicate that the temporal pattern of these isoforms differ significantly between sustaining pregnancies and these which will fail.

This early pregnancy hCG isoform declines rapidly, being replaced by the presumably less highly branched hCG glycoform of a mature pregnancy (Kovalevskaya et al. 
1999a). The replacement of the early hCG isoform(s) by those of later pregnancy is a continuous process. Both families of hCG isoforms appear to be present throughout pregnancy, but at substantially different concentrations. Since no one hCG assay is $100 \%$ specific for a particular hCG isoform we have developed a method to present the relative amounts of hCG isoforms as a ratio of hCG concentrations measured by two assays. One has primary affinity for early pregnancy hCG (B152-B207*, asterisk means radiolabelled detection antibody) and the other (B109-B108`) has primary affinity to the later isoform(s). We believe that the ratio of the two assays most accurately reflects the continuous transition of hCG isoforms as pregnancy progresses. The present paper expands these studies to characterize the expression of a family of putative hyperglycosylated hCG isoforms in in vitro fertilization (IVF) normal pregnancy and IVF early pregnancy loss (EPL) as well as throughout normal pregnancy compared with later miscarriages.

Although it was reported earlier that B152 is directed to carbohydrate and peptide backbone structure, characterization of its recognition patterns was not completed. For example, it appeared that both $\mathrm{N}$-linked as well as O-linked carbohydrates may be involved in the recognition site (Birken et al. 1999). Furthermore, it was assumed that B152 was a conformation-directed antibody. In the following studies we report that B152 is directed to a linear epitope within the $\beta \mathrm{COOH}$-terminal region (CTP) of hCG or hCG free $\beta$ subunit (hCG $\beta$ ).

\section{Materials and Methods}

\section{Patient samples}

Set no. 1. Urine samples from patients undergoing ovum donation The program for oocyte donation was reviewed and approved by the Institutional Review Board and the Medical Ethics Committee of Columbia University, College of Physicians and Surgeons, Columbia-Presbyterian Medical Center.

Upon completion of required pre-screening examinations and matching with an approved donor, oocyte donors underwent ovarian hyperstimulation with human menopausal gonadotropins and were monitored by transvaginal ultrasound and serum estradiol levels. When follicle size reached 18-20 mm, hCG was given to trigger ovulation. Thirty-six hours later, transvaginal oocyte aspiration was performed under ultrasound guidance. Recipients were synchronized to an oocyte donor using a standard regimen of hormone replacement consisting of oral micronized estradiol and intramuscular progesterone, as previously described (Sauer et al. 1995). Recipients with residual ovarian function underwent pituitary down-regulation with a gonadotropin-releasing hormone agonist to render them functionally agonadal prior to initiating hormone replacement.
Following fertilization, three to five embryos were transcervically transferred at $72 \mathrm{~h}$ to the recipient's uterus. Pregnancy was confirmed by serum hCG $\beta 9$ and 12 days following embryo transfer (ET). Prescribed hormonal replacement was continued through 12 weeks of gestation.

Twenty-two IVF donor egg recipients who underwent an ET without pretreatment with exogenous hCG (i.e. donor egg) collected urine every morning starting the day after ET. A total of 542 samples was collected and analyzed in this study. The ten patients who did not have detectable hCG (presumably because the embryo failed to implant into the endometrium) were excluded from the analysis.

Twelve women were positive in the hCG assays (399 samples). Five of the twelve subjects had EPL, four had a singleton pregnancy and three had multiple fetuses (two or three).

For those twelve patients who had a positive value of hCG $(>2 \times$ least detectable dose) the range of the collection period was 7-48 days with a median collection duration of 34 days. One hCG-positive patient collected urine during days 1 to 20 and 39 to 60 . The maternal age in this group of patients was in the range 33-50 years with a median of $42 \cdot 8$ years.

Two urine samples $(50 \mathrm{ml}$ each) from the same pregnant woman (43 years old) who underwent IVF procedure were used for immunoblotting. One sample was collected at the 31st day post-ET, the second at the 45th day post-ET.

Set no. 2. Normal pregnancy urine samples Two hundred and fifteen samples from ten normally conceived pregnancies were collected at an interval of 1-2 weeks throughout complete pregnancies (i.e. terminated in a live birth) and were compared with eleven miscarriages (37 samples). Sample collection started from weeks 3 to 9 (median 6.13 weeks) from the last menstrual period (LMP).

Urine samples were collected at Columbia-Presbyterian Medical Center under an Institutional Review Boardapproved protocol.

\section{Materials}

Antibodies B152 and B151 were developed to a choriocarcinoma hCG (C5) (Birken et al. 1999), while B201 and B207 are directed to hCG $\beta$ core fragment (hCG $\beta c f$ ) and hCG $\beta$ (Krichevsky et al. 1988, O'Connor et al. 1988). CTP103, developed to synthetic peptide $\beta$ residues 109145 (Krichevsky et al. 1994a) was described earlier. Anti-mouse IgG antibody was from DAKO (Carpinteria, CA, USA). Sequencing grade modified trypsin (13 U/ $\mathrm{gg})$ was obtained from Promega (Madison, WI, USA). ECL Western blot reagents and Hyperfilm-ECL were purchased from Amersham International (Amersham, Bucks, UK). Polyvinylidene difluoride membrane (PVDF) and other materials related to electrophoresis and 
electrophoretic transfer were obtained from Bio-Rad (Richmond, CA, USA). Other reagents were from Fisher (Pittsburg, PA, USA).

The non-nicked hCG $\beta$ (preparation 863), isolated from the CR127 preparation of hCG, was used in immunoblottings (Birken et al. 1993). Choriocarcinoma hCG C5 (designated 673 in this report), a 100\% nicked hCG having extra carbohydrate moieties, purified from the urine of a choriocarcinoma patient (Elliott et al. 1997), was supplied by Dr L Cole (University of New Mexico, NM, USA). Hormones used for B151-B152^ assay crossreactivity characterization were: hCG $\mathrm{C} 5$ (preparation (prep.) 510), hCG C5 free $\beta$ (prep. 544), hCG nicked (prep. 656), hCG CR129, hCG $\beta$ CR117, hCG $\beta$ cf (prep. 455), hCG $\alpha$ CR119.

\section{Immunoradiometric assays}

Immunometric assay construction has been described previously (O'Connor et al. 1988). The methodology used to analyze urine samples has also been completely described (Kovalevskaya et al. 1999a). It is based on the combination of two hCG solid-phase immunometric assays: B109$\mathrm{B} 108^{\star}$ and B152-B207*. Intra-assay variation was 3\% for B109-B108* assay and 5\% for B152-B207* assay, interassay variation was $10 \%$ for B109-B108* assay and $11 \%$ for B152-B207^ assay. Sensitivity (least detectable dose) defined as +2 s.D. from the zero calibrator, was $1 \mathrm{fmol} / \mathrm{ml}$ for the B109-B108* assay, $4 \mathrm{fmol} / \mathrm{ml}$ for the B152-B207* assay and $11 \mathrm{fmol} / \mathrm{ml}$ for the B151-B152* assay.

Urinary non-nicked hCG was purified from the CR127 preparation of pooled normal pregnancy hCG and was used as a standard in both B109-B108* and B152-

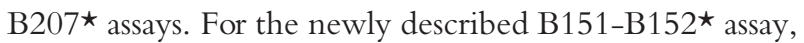
hCG C5 was used as a standard and $10 \mu \mathrm{g} / \mathrm{ml} \mathrm{B} 151$ was used for microtiter plate coating.

\section{Statistical analysis}

Set no. 1. Urine samples from patients undergoing ovum donation For statistical analysis, the trajectory of the daily log-transformed B152/B109 ratio from the day following ET (first analysis) or from the 1st day of detectable hCG (second analysis) through the 30 succeeding days were compared between the IVF normal pregnancy group and the IVF EPL group using mixed-effects models (Laird \& Ware 1982). Based on graphical inspection of outliers, mean ratio trajectories and their distributions, the ratio was log transformed to improve linear time trend and normality. The mixed-effects model was used because (a) heterogeneity of an individual trajectory can be modeled through a random slope representing the time trend of the ratio, (b) serial correlation within repeated measures of the log-ratio can be modeled, and (c) incomplete data due to pregnancy loss (i.e. unequal number of sampling points) can be used, thus avoiding any imputation and utilization of all available data. To capture the observed time trend, we divided the sampling period into time intervals (e.g. 1 st to 5 th days, 5 th to 10 th, 10 th to 15th, 15th to 20th and so on), and employed the piecewise regression method (Montgomery \& Peck 1982) so that the differential time trend within each time interval can be modeled. Significant interaction of the time trend for a given interval and experimental group implies a differential time trend between two groups within the time interval. Although the mixed-effects modeling approach permits us to model inter- and intrapatient variability based on incomplete data, we are aware of the limitation of statistical inference based on a relatively small sample size including lack of statistical power and generalizability of findings.

Set no. 2. Normal pregnancy urine samples Logtransformed measurement profiles (log B152/B109 ratio, $\log \mathrm{B} 152, \log \mathrm{B} 109)$ were analyzed by the mixed-effects model consisting of random intercept, random slope against gestational age of subject, outcome (i.e. pregnant and spontaneous abortion) and interaction between time trend (i.e. slope) and outcome.

\section{Partial purification of early pregnancy urine samples prior to} SDS-PAGE

Two specimens $(50 \mathrm{ml}$ each) were obtained from pregnant woman at gestation days 31 and 45 from ET who underwent IVF procedures. The specimens were adjusted to neutral $\mathrm{pH}$ with $1 \mathrm{M}$ Tris- $\mathrm{HCl}$ buffer $(\mathrm{pH} 8 \cdot 0)$ and allowed to precipitate overnight at $4{ }^{\circ} \mathrm{C}$. The supernatant was filtered through a 0.45 micron filter (Nalgene, Rochester, NY, USA) and concentrated in a 3000 cutoff Centricon ultrafilter YM-3 (Amicon, Danvers, MA, USA) to $3 \mathrm{ml}$. Each urine concentrate was desalted on a Sephadex G-25 column (Pharmacia, Piscataway, NJ, USA) in 0.05 $\mathrm{M}$ ammonium bicarbonate and then lyophilized. The dried powder was suspended in 1-2 $\mathrm{ml}$ column buffer $(0.05 \mathrm{M}$ ammonium bicarbonate) and then sizefractionated on Superose $12(30 \mathrm{~cm} \times 1 \mathrm{~cm}$; Pharmacia $)$ tandem columns (two in series). Fractions eluting where the hCG standard eluted were pooled and lyophilized. These fractions were used for the SDS gel electrophoresis and blots.

\section{Separation of subunits of C5 (673)}

Fifty micrograms hCG C5 were incubated in $4 \mathrm{M}$ guanidine acetate, $\mathrm{pH} 4$ for $2 \mathrm{~h}$ at $37^{\circ} \mathrm{C}$, and the dissociated subunits were separated on a Pharmacia Smart system using a uRP C2/C18 PC 3·2/3 column. Column buffers were $0 \cdot 1 \%$ trifluoroacetic acid (TFA) in water (A) and the $0 \cdot 1 \%$ TFA in $100 \%$ acetonitrile (B); flow rate was $0.24 \mathrm{ml} /$ min; gradient: $0 \% \mathrm{~B} 10 \mathrm{~min} ; 50 \% \mathrm{~B}$ at $40 \mathrm{~min}$, linear 
Table 1 Comparison of the log-transformed B152/B109 ratio time trend in IVF early pregnancy and IVF early pregnancy loss (EPL) urine samples (from the day after embryo transfer $(\mathrm{ET}))$

\begin{tabular}{|c|c|c|c|c|}
\hline & \multicolumn{2}{|c|}{$\begin{array}{l}\text { All IVF normal pregnancy } \\
\text { vs IVF EPL }\end{array}$} & \multicolumn{2}{|c|}{$\begin{array}{l}\text { IVF normal pregnancy (single fetus) } \\
\text { vs IVF EPL }\end{array}$} \\
\hline & $F(d f, d f)$ & $P$ value & $\mathrm{F}(\mathrm{df}, \mathrm{df})$ & $P$ value \\
\hline \multicolumn{5}{|c|}{$\begin{array}{l}\text { Time interval } \\
\text { (days from the } \\
\text { day after ET) }\end{array}$} \\
\hline $5-10$ & $4 \cdot 28(1,263)$ & 0.0395 & $8 \cdot 76(1,179)$ & 0.0035 \\
\hline $10-15$ & $7 \cdot 21(1,263)$ & 0.0077 & $8 \cdot 32(1,179)$ & 0.0044 \\
\hline $15-20$ & $6 \cdot 37(1,263)$ & $0 \cdot 0122$ & $8 \cdot 76(1,179)$ & 0.0035 \\
\hline $20-25$ & $0 \cdot 74(1,263)$ & $0 \cdot 3900$ & $1 \cdot 80(1,179)$ & $0 \cdot 1815$ \\
\hline
\end{tabular}

gradient. The nicked $\beta$ peak elutes prior to $\alpha$ and both peaks were separately pooled and dried.

Reduction and alkylation of $h C G \beta$ and choriocarcinoma $h C G$ C5 free $\beta$ subunit

Lyophilized hCG C5 free $\beta$ subunit $(20 \mu \mathrm{g})$ and hCG $\beta$ preparation $863(87 \mu \mathrm{g})$ were reduced, alkylated, and separated on reverse phase HPLC as described earlier (Birken et al. 2001).

\section{SDS-PAGE, Western blot and dot-blot analysis}

SDS gels were run according to the procedure of Laemmli (1953). Proteins in the gel were transferred electrophoretically according to the directions provided by the manufacturer (Bio-Rad). Blots were visualized by autoradiography according to the manufacturer's directions (Amersham ECL reagent and Kodak Hyperfilm-ECL).

\section{Liquid phase radioimmunoassay competition studies}

Liquid phase radioimmunoassay was conducted as described (Birken et al. 1999). Preparation of all competitor forms of hCG have been described earlier (Rosa et al. 1984, Puett \& Birken 1989, Birken et al. 1991, 1993, 2000). Freshly made water solutions (calibrated in molarity by amino acid analysis) were diluted with carrier protein-containing buffer.

\section{Protein determination}

Hormone concentrations were determined by amino acid analysis on a Beckman 6300 according to the manufacturer's instructions.

\section{Creatinine}

Urine hCG values were normalized to creatinine, measured in a 96-well microtiter plate format by a procedure adapted from Taussky (1954).

\section{Results}

Analysis of daily urine collection from IVF patients (set no. 1) The apparent values of hCG (normalized to creatinine) in the B152 assay were higher than in the B109 assay in both IVF normal pregnancy and IVF losses (Fig. 1). However, the proportion of each isoform presented as the B152/ B109 ratio changed significantly both as a function of gestational age and pregnancy outcome. As shown in Fig. 1, in normally continuing IVF early pregnancy the B152/B109 ratio declined rapidly as pregnancy progressed (Fig. 1 insert C1). In contrast, the B152/B109 ratio exhibited a more shallow decline in IVF EPL (Fig. 1 insert D1). Table 1 presents the outcome of the statistical analysis of the B152/B109 ratio commencing with the day after ET. It was determined that, for both single fetus pregnancies and single and multiple fetuses combined, significant differences of the B152/B109 ratio were observed between the IVF normal pregnancy and IVF pregnancy loss group. These differences maintained significance throughout days 5-20 from the day after ET.

Upon assaying the urine samples which had been started from the day after ET we found that the day of first detection of a positive hCG value in urine varied. The mean day of the first detection was 6.75 days from ET (range was 5-10 days). When the time trend analysis commenced with the first day of detectable hCG, a statistically significant difference in the B152/B109 ratio between IVF normal pregnancy for both multiple and singleton fetuses analyzed together and IVF EPL was only approached $(P=0.058)$ in the time interval of the 10th to the 15th days (Table 2). There was, however, a significant difference in the 5- to 10-day and the 10- to 15-day intervals between IVF normal pregnancy (single fetus) and IVF EPL.

There was no statistically significant difference in maternal age between the IVF normal pregnancy group (mean was 45.3 years) and the IVF EPL group (mean was $41 \cdot 5$ years $)(P=0 \cdot 242)$. 

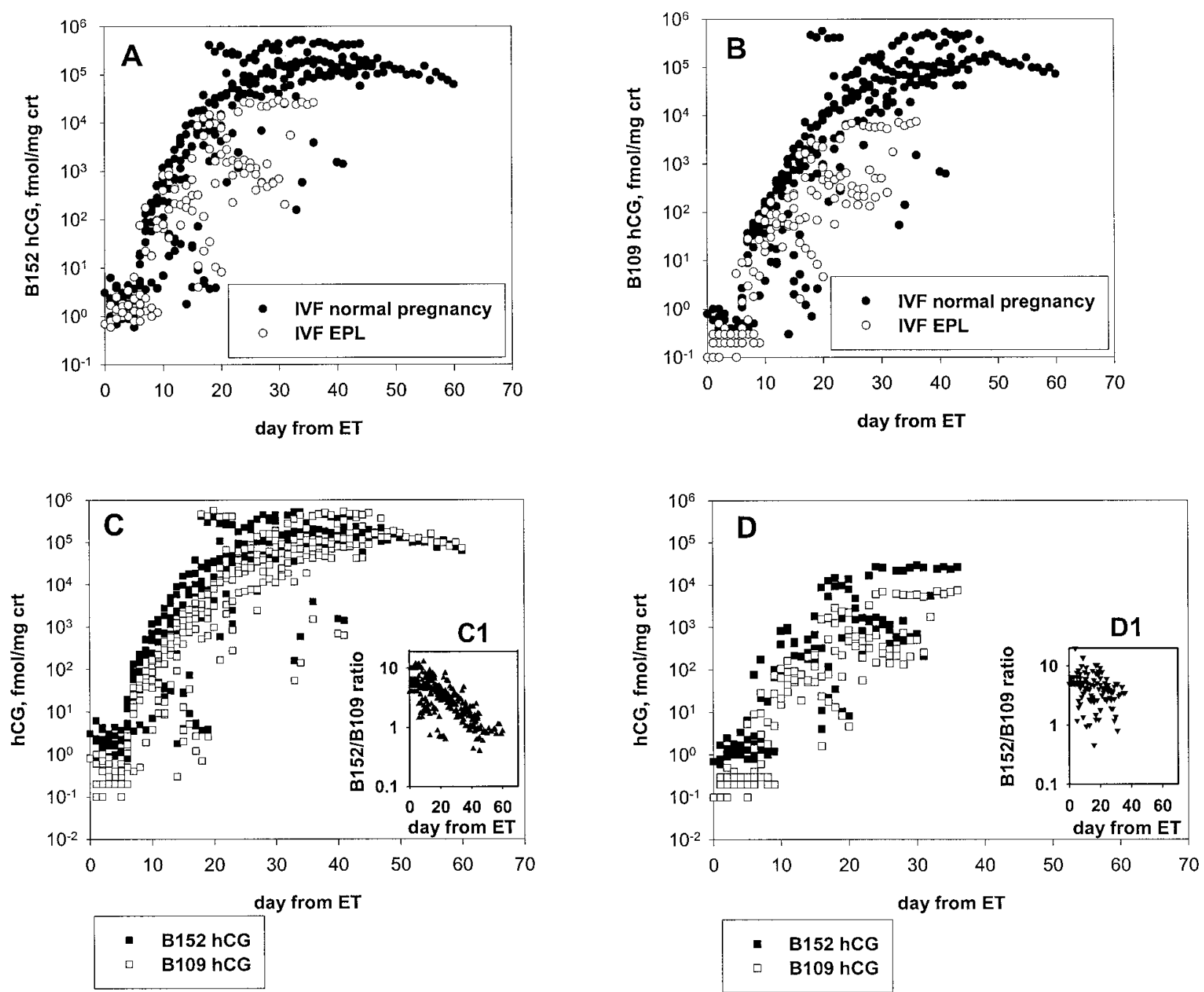

Figure 1 hCG isoform profiles in IVF patients (normal pregnancy and EPL) urine. hCG was measured by the B152-B207* assay (A) and by the B109-B108* assay (B); hCG isoforms (C) and the B152/B109 ratio (insert C1) in normal IVF pregnancy; hCG isoforms (D) and the B152/B109 ratio (insert D1) in IVF EPL. Note that the ratio in IVF EPL (insert D1) has a shallow slope as compared with that of IVF continuing pregnancy (insert $\mathrm{C} 1$ ). crt, creatinine.

Analysis of weekly collections through normal pregnancy and spontaneous abortions (set no. 2)

The B152/B109 ratio profiles for naturally conceived normal pregnancy and miscarriages throughout pregnancy are presented in Fig. 2.

Statistical data analysis revealed a significant differential time trend between the profiles of normal pregnancies and spontaneous abortion on $\log (\mathrm{B} 152 / \mathrm{B} 109$ ratio) $[\mathrm{F}(1,62)=4 \cdot 25 P=0 \cdot 043]$. The $\mathrm{B} 152 / \mathrm{B} 109$ ratio declined with gestational age but the rate of decline was faster in the spontaneous abortion case than in normal pregnancy. Levels of the B109 hCG isoform became progressively higher in the normal pregnancy group as compared with the loss group $[\mathrm{F}(1,62)=6 \cdot 89 P=0 \cdot 011]$.
Construction, characterization and application of immunometric assay B151-B152* specific for C5 (choriocarcinoma hCG)

This immunoassay was constructed using monoclonal antibody B151 as a capture and monoclonal antibody B152 for detection. The B151 antibody recognizes the nicked epitope of C5 as well as the nicked epitope on hCG from normal pregnancy (Birken et al. 1999, Kovalevskaya et al. 1999b). The B152 antibody recognizes a hyperglycosylated epitope of C5 and a putatively similar structure present in an early pregnancy hCG isoform (Birken et al. 1999, Kovalevskaya et al. 1999a). The B151-B152^ assay, which recognizes C5 choriocarcinoma hCG (nicked hyperglycosylated hCG), is quite specific. Cross-reactivity with nicked hCG is $9 \%$, with hCG (CR129) it is $2 \%$, with 
Table 2 Comparison of the log-transformed B152/B109 ratio time trend in IVF early pregnancy and IVF EPL urine samples (from the 1 st day of hCG detection)

All IVF normal pregnancy vs IVF EPL

$\overline{\mathrm{F}(\mathrm{df}, \mathrm{df}) \quad P \text { value }}$

Time interval

(days from the first hCG detection)

$1-5$

$5-10$

$10-15$

$15-20$

$\begin{array}{ll}1 \cdot 16(1,214) & 0 \cdot 2819 \\ 2 \cdot 57(1,214) & 0 \cdot 1104 \\ 3 \cdot 63(1,214) & 0 \cdot 0582 \\ 1 \cdot 88(1,214) & 0 \cdot 1714\end{array}$

IVF normal pregnancy (single fetus) vs IVF EPL

$\overline{\mathrm{F}(\mathrm{df}, \mathrm{df}) \quad P \text { value }}$

hCG C5 free $\beta$ subunit it is $1 \%$ and cross-reactivity with all other tested analytes (hCG $\beta$, hCG $\beta c f, h C G \alpha$ ) is under $1 \%$.

On the basis of the ratio of hCG isoforms detected by the B152-B207* and B109-B108* assays for intact hCG we found a similarity between early pregnancy hCG and choriocarcinoma hCG C5 (Kovalevskaya et al. 1999a). When we used B151 as a capture and analyzed early pregnancy urine samples in the B151-B152* assay we did not detect a C5-similar hCG to any appreciable extent. The conclusion was that early pregnancy hCG is

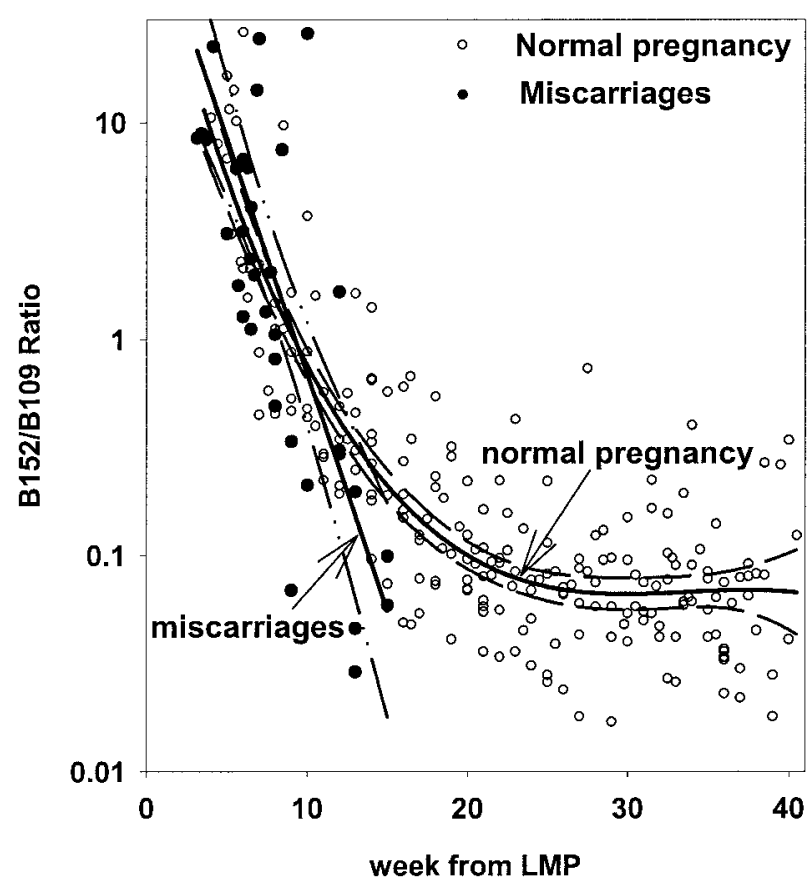

Figure 2 B152/B109 ratio throughout pregnancy. Urine samples were collected from ten normally pregnant patients (215 samples) and from eleven patients with miscarriages (37 samples) weekly. Regression curve and 95\% confidence interval are shown for normal pregnancy $\left(r^{2}=0 \cdot 76\right)$ and for miscarriages $\left(r^{2}=0 \cdot 57\right)$.

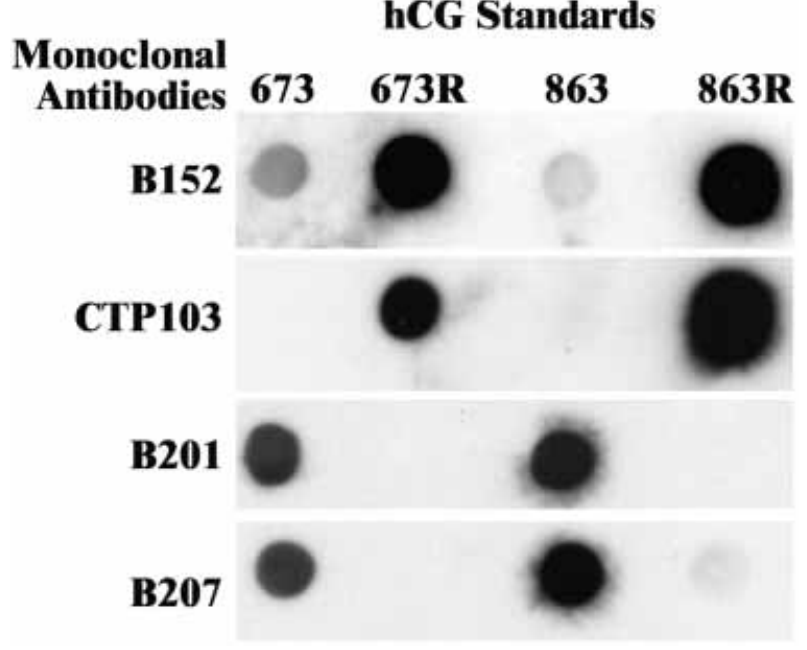

Figure 3 Comparison of B152 immunoreactivity towards non-reduced and reduced forms of choriocarcinoma hCG C5 (673) and non-nicked hCG $\beta$ (863) with monoclonal antibodies CTP103, B201 and B207. R indicates reduced forms of 673 and 863.

not nicked, in contrast to C5 which is 100\% nicked. Immunoblot experiments are described below which also support this finding.

Characterization of the B152 epitope by Western blot analysis and by competitive liquid phase immunoassay

Dot blots were used to compare four different antibodies by the relative intensity of immunostaining of reduced and non-reduced hCG $\beta$ and choriocarcinoma hCG C5 (Fig. 3). Two antibodies known to require a conformational epitope were compared with one antibody made to a synthetic peptide that was directed to a linear determinant in the $\beta-\mathrm{COOH}$ region (CTP). The conformationaldirected antibodies, B201 and B207, were both generated to the hCG $\beta c$, and both also recognize hCG $\beta$ (Krichevsky et al. 1988, 1994b). The linearly directed antibody CTP103 was made to a synthetic peptide 


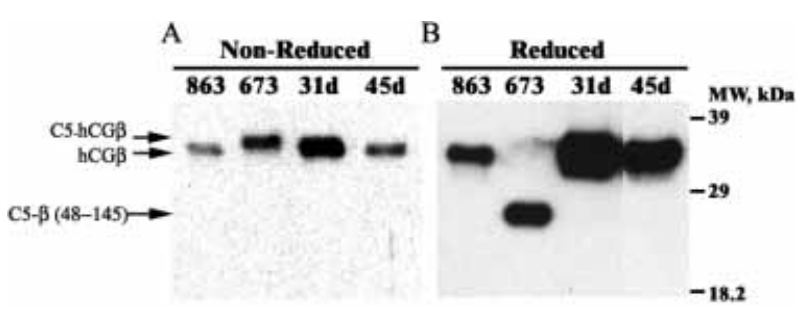

Figure 4 Comparison of gel electrophoretic migration sizes of the hCG $\beta$ subunits from IVF patient urine from gestational ages of 31 st day (31d) and 45th day (45d) with the choriocarcinoma hCG C5 (673) and normal pregnancy hCG $\beta$ (863) by immunoblot analysis with B152. Samples were prepared in non-reduced (A) or reduced (B) conditions. The size of early pregnancy urine hCG $\beta$ resembles that of standard hCG $\beta$ (863) by this technique rather than that of choriocarcinoma hCG $\beta$ from C5 (673). Upon reduction, only $\beta_{48-145}$ (as identified by using purified peptide in Fig. 3) stains with B152.

composed of residues 109-145 of hCG $\beta$ and appears to bind this mobile region of hCG not visible on the crystal model of hCG (Krichevsky et al. 1994a, Lapthorn et al. 1994, Wu et al. 1994). It is clear from Fig. 3 that B201 and B207 stain non-reduced hCG $\beta$ (863) and non-reduced hCG C5 (673) much better than reduced forms of these same molecules. The choriocarcinoma hCG C5 was used for the generation of B152 (Birken et al. 1999). In contrast, B152 and CTP103 bind much better to reduced hCG $\beta$ and reduced choriocarcinoma hCG C5 as compared with non-reduced forms. Thus, B152 most closely resembles the binding properties of antibody CTP103 which is known to be directed to a linear epitope.

\section{Examination of the isoforms recognized by $B 152$ in early} pregnancy urine

Choriocarcinoma hCG C5 was reported to be hyperglycosylated at both its $\mathrm{N}$ - and O-linked carbohydrate moieties (Elliott et al. 1997). Extra carbohydrate makes this hCG isoform migrate slower in SDS gels than standard late first trimester pregnancy hCG. Likewise, if early pregnancy hCG isoforms are similarly hyperglycosylated, they too should migrate more slowly. All samples were boiled in SDS so we were visualizing dissociated $\beta$ chains. Figure 4A shows that choriocarcinoma C5 hCG $\beta$ (from 673 ) is only slightly larger than the standard hCG $\beta$ (863) on these blots but that the 31st day hCG $\beta$ (rich in the $\mathrm{B} 152 \mathrm{hCG}$ isoforms as determined by measurement of a B152/B109 ratio of 3.4) as well as the 45th day hCG $\beta$ (lower in the B152 isoform as indicated by a B152/B109 ratio of 0.5$)$ are both similar in migration position to standard mid-pregnancy hCG $\beta$ (863). Reduction of these samples by mercaptoethanol also did not show a migration difference between standard hCG $\beta$ and the forms of hCG $\beta$ in 31 st day and 45 th day gestation urine (Fig. $4 \mathrm{~B}$ ). It was noted on these blots of reduced samples that no nicked forms of $\beta$ were stained except for the nicked $\beta$

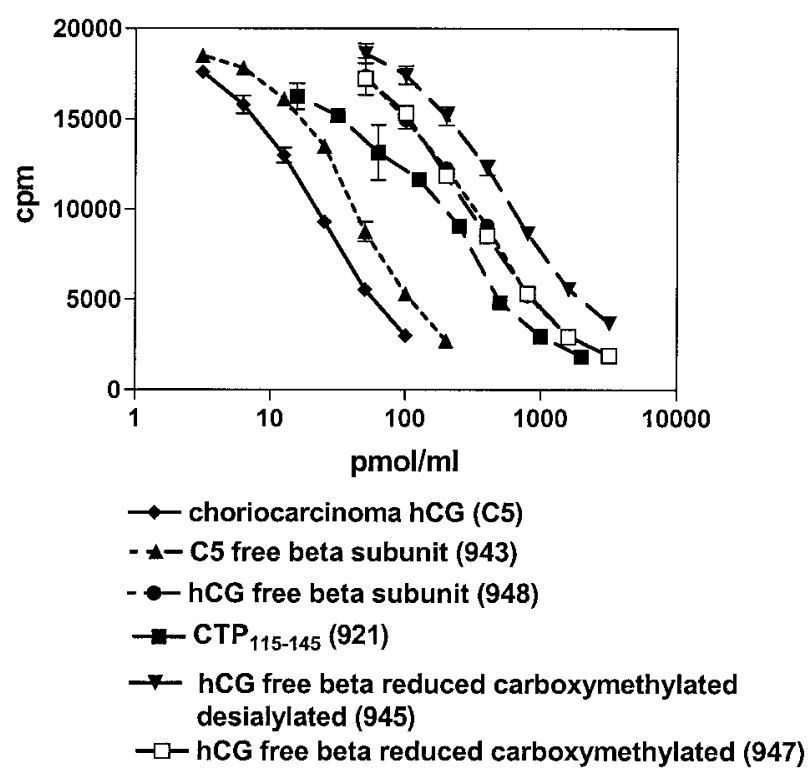

Figure 5 Liquid phase radioimmunoassay competition study. hCG C5 (choriocarcinoma hCG, immunogen for B152) was radiolabeled and liquid phase competition curves were developed by use of solutions of each hCG-related competitor calibrated by amino acid analysis in molarity. Competitors as noted on the Figure: hCG C5, hCG C5 free $\beta$ subunit, free $\beta$ 115-145 natural peptide, reduced carboxymethylated hCG free $\beta$, hCG $\beta$, and desialylated hCG $\beta$.

subunit present in choriocarcinoma hCG C5 (673). Reduction greatly increased the intensity of immunoblot staining of hCG isoforms, as shown by the dot blots of Fig. 3, making it difficult to discern differences in band positions of the reduced isoforms in Fig. 4.

The $\beta$ subunit of nicked hCG, e.g. hCG C5, can be cleaved into two halves by simply reducing the disulfide bridges. Separation of reduced hCG C5 on SDS gels separates the $\mathrm{N}$-glycosylated amino terminal half from the $\mathrm{O}$-glycosylated $\mathrm{COOH}$ terminal half. In reduced hCG C5 (673), antibody B152 (Fig. 4B) as well as CTP103 (not shown) recognize a single band at approximately $20 \mathrm{kDa}$ molecular weight, and we assumed this band is $\beta_{48-145}$. Preparation of purified, reduced and alkylated $\beta_{48-145}$ confirmed its staining by B152. However, this does not prove that $\mathrm{B} 152$ can recognize the $\mathrm{COOH}$-terminus $\left(\beta_{115-145}\right)$ alone.

Therefore, we performed a series of liquid phase radioimmunoassay studies using radiolabeled hCG C5 as a tracer and a variety of hCG isoforms as competitors (Fig. 5). This study demonstrated that hCG C5 is the most potent competitor in liquid phase with itself as tracer as one would expect, since it is the immunogen for antibody B152. Free C5 $\beta$ was the next most potent. The other reagents were derived from standard pregnancy hCG, which is not hyperglycosylated, resulting in their lower potencies when displacing labeled hCG C5 from B152. 


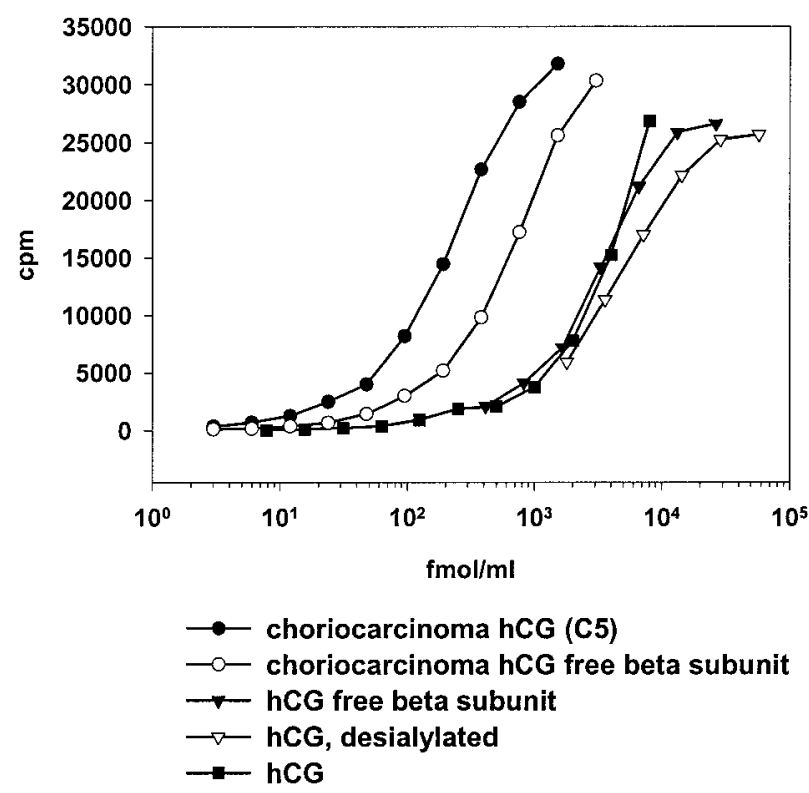

Figure 6 Binding curves for hCG-related molecules in the solid phase two-site immunometric assay B152-B207*.

However, it is clear that hCG $\beta$ and reduced, carboxymethylated hCG $\beta$ (RCM $\beta$ ) which is a permanently denatured, linearized molecule, are equipotent, demonstrating that the $\mathrm{B} 152$ does not require a conformational epitope. In addition, the similar potencies of free $\beta_{115-145}$ indicate that most or all of the B152 epitope is in this region. Removal of sialic acid from RCM $\beta$ diminishes its potency somewhat but it can still compete, demonstrating that sialic acid is a minor component of the epitope of B152.

These studies by liquid phase competition were further supported by direct comparisons of calibrated reference solutions using the B152-B207* two-site assay (Fig. 6). The solutions, molarity calibrated by amino acid analysis, were hCG, hCG C5, hCG C5 $\beta$, hCG $\beta$ and desialylated hCG. The comparative binding curves agreed well with the liquid phase competition curves of Fig. 5 (except that reduced and alkylated and free CTP solutions could not be tested in the B152-B207* assay since they are not recognized by the detection antibody). hCG C5 is the highest affinity standard followed closely by its free $\beta$ subunit. All other reference solutions bind with lower affinity and desialylated hCG binds very similarly to that of naturally sialylated hCG.

\section{Discussion}

hCG isoforms in early and later pregnancy urine

The existence of hCG glycoforms has been documented for some time (Fein et al. 1980, Wide \& Hobson 1987,
Skarulis et al. 1992, Nemansky et al. 1998). Most of this work was either based on isoelectric focusing patterns which are not feasible for general measurements or else by carbohydrate analysis on a few specimens. All current hCG immunoasays participate in the recognition of these isoforms to an undetermined extent since standard preparations of hCG glycoforms have not been available. We have developed the first immunometric system capable of quantifying the relative content of early pregnancy isoforms as compared with late first trimester hCG isoforms (Kovalevskaya et al. 1999a). Our methodology allows for the measurement of two isoform families recognized by their differential affinity for the antibodies used in the construction of two immunometric assays. One of the assays (B152-B207^) has primary affinity for the hyperglycosylated hCG isoform which predominates in very early pregnancy (first 6 weeks). The second assay (B109$\left.\mathrm{B} 108^{\star}\right)$ has primary affinity for the hCG isoforms of later pregnancy. The ratio of values obtained in these two assays allows us to visualize an immunochemical profile of the progression of hCG isoforms throughout pregnancy.

As can be observed from Fig. 1C, the B152 hCG isoform rises rapidly in IVF early normal pregnancy, preceding the increase of the B109 hCG isoform. Figure 1 (insert C1) presents the ratio of the two isoforms, showing the shift in the relative proportion of the two isoforms as gestation proceeds. Figure 1D presents the apparent hCG isoform concentrations in IVF EPL and Fig. 1 (insert D1) illustrates the B152/B109 ratio in IVF EPL. The original analysis of the data started from the day of ET (Table 1), and resulted in an improved discrimination over that when the data were analyzed from the first day of positive hCG detection in both assays (Table 2).

It is important to note that elimination of these first points in the latter approach had the effect of diminishing an observed difference between the IVF continuing pregnancy group and the IVF loss group - namely that the loss ratio profile (log ratio vs time) lags behind that of the continuing pregnancy group, i.e. if the loss group ratio is shifted forward by approximately 5 days the profiles assume greater similarity.

The observed decrease in the power of discrimination between the IVF pregnancy and IVF EPL groups consequent to the two statistical approaches (i.e. analysis from day of ET vs day of first hCG detection) appears to be, at least in part, due to the partial loss of the lag time observed in the IVF EPL group as compared with the IVF pregnancy group as a result of the normalization of the two profiles to the day of hCG detection. The profiles of the two groups (log ratio vs time) become roughly parallel when this frame shift is eliminated. It appears that the biological activity or transformation, perhaps cytotrophoblast cell differentiation, which is signified by the change in isoform ratio, can be at least partially accounted for by its occurrence at a later time in EPL gestations. 
Our prior studies of hyperglycosylated hCG excretion throughout pregnancy (103 samples at irregular intervals) indicated an inflection point in the regression curve around 29 weeks of gestation (Kovalevskaya et al. 1999a, Fig. 2). The present study, with more samples (215 from ten subjects) at regular intervals, fails to show this inflection. The B152/B109 ratio decreases to low levels after 22 weeks of gestation and remains essentially constant until birth (Fig. 2).

Although significant differences in the behavior of the hCG isoform ratio between clinical spontaneous abortion and naturally conceived normal pregnancy were found, the pattern of change did not mimic or coincide with that of IVF EPL. This may indicate that different cellular factors controlling the expression of hCG isoforms are operative as gestation proceeds.

\section{C5-like hCG in early pregnancy urine}

In a previous study we reported that nicked hCG is not a significant constituent of early pregnancy urine (Kovalevskaya et al. 1999b). For that study we used the B151-B604* assay which recognizes nicked hCG originating from normal pregnancy hCG. Now we have constructed the B151-B152^ assay which specifically recognizes choriocarcinoma hCG C5 (100\% nicked). Both B151 and B152 antibodies were developed to choriocarcinoma hCG C5 (Birken et al. 1999). We applied this assay to IVF early pregnancy urine and did not detect hCG by this assay.

Comparison of results from the B151-B152* and the B152-B207* assays allows us to conclude that the similarity between early pregnancy hCG and choriocarcinoma hCG C5 is not due to nicking but to hyperglycosylation.

\section{Epitope recognized by $B 152$}

We used B152 as a capture antibody in a two-site assay and a common, anti-native hCG $\beta$ antibody as the detection antibody. The detection antibody, B207, is clearly directed to a conformational determinant as shown by the dot-blot analysis (Fig. 3). B152 binds preferentially to reduced, denatured hCG $\beta$ (Fig. 3) and binds specifically to the COOH-terminal half of the hCG $\beta$ (Fig. 4). Liquid phase competition assays using radiolabeled hCG C5 as a tracer indicate that B152 is directed to a linear epitope since it binds to reduced and alkylated hCG $\beta$. In liquid phase assay, B152 recognized hCG $\beta$ and reduced and alkylated hCG $\beta$ equivalently. This result differs from the blot analysis, which showed better recognition of the reduced hormone form. Presumably this is due to additional changes induced by binding of the reduced protein to the membrane surface. The liquid phase studies also showed that free $\beta_{115-145}$ competed as well as hCG $\beta$, proving that B152 is a linearly directed antibody with all or most of its binding within this CTP region.
Conformationally directed hCG $\beta$ antibodies did not bind to alkylated hCG $\beta$ to a significant extent in either liquid or solid phase immunoassays. Liquid phase radioimmunoassay showed that the extent of sialylation did not affect B152 binding significantly. These findings were further substantiated by examining binding of the amino acid analysis-calibrated hormone solutions by the B152-B207* two-site assay. The lack of the effect of sialic acid content was substantiated. Our earlier report demonstrated that carbohydrate was part of the B152-recognized site by the use of hCG isoforms which were not hyperglycosylated but identical in all other respects (Birken et al. 1999).

Antibody B152 was made to a choriocarcinoma form of hCG which exhibited both hyperglycosylated N- and O-linked carbohydrate moieties. In our earlier report it was not clear if both types of carbohydrates were involved in the B152 epitope and this determinant was presumed to be conformational (Birken et al. 1999). The studies reported here have shown that the determinant is linear and similar to that $\mathrm{COOH}$-terminal part of hCG $\beta$ which is recognized by CTP antibodies, except for its preference for hyperglycosylated O-linked carbohydrate groups. (The O-linked glycans of hCG $\beta$ are located at serine residues $121,127,132$, and 138.)

The O-linked glycosylation process appears altered in the trophoblast cells in early placenta, resembling the type of sugar synthetic pathway operative in trophoblastic malignancy. Although many types of cancerous cells tend to synthesize truncated O-glycans rather than more complex types, this is not the case for trophoblastic malignancies (Brockhausen 1999, Kobata \& Takeuchi 1999). hCG-secreting malignancies display more complex glycans, some of which are not sialylated or reduced in sialic acid content compared with normal pregnancy hCG.

\section{Acknowledgements}

This work was supported by grant ES07589 from the NIEHS and the Office of Research on Women's Health, $\mathrm{NIH}$, grant RR00645 from NCRR, NIH, and grant AG13783, NIH. The authors express their appreciation to Dr Cole (University of New Mexico) for providing choriocarcinoma hCG C5. We gratefully acknowledge the contribution of Alana Shear and Rebecca Dean (Columbia-Presbyterian Medical Center) in the performance of IVF specimen collection.

\section{References}

Birken S, Gawinowicz MA, Kardana A \& Cole LA 1991 The heterogeneity of human chorionic gonadotropin (hCG). II. Characteristics and origins of nicks in hCG reference standards. Endocrinology 129 1551-1558.

Birken S, Chen Y, Gawinowicz MA, Lustbader JW, Pollak S, Agosto G, Buck R \& O'Connor J 1993 Separation of nicked human 
chorionic gonadotropin (hCG), intact hCG, and hCG beta fragment from standard reference preparations and raw urine samples. Endocrinology 133 1390-1397.

Birken S, Krichevsky A, O'Connor J, Schlatterer J, Cole L, Kardana A \& Canfield R 1999 Development and characterization of antibodies to a nicked and hyperglycosylated form of hCG from a choriocarcinoma patient: generation of antibodies that differentiate between pregnancy hCG and choriocarcinoma hCG. Endocrine $\mathbf{1 0}$ 137-144

Birken S, Maydelman Y \& Gawinowicz MA 2000 Preparation and analysis of the common urinary forms of human chorionic gonadotropin. Methods 21 3-14.

Birken S, Gawinowicz MA, Maydelman Y \& Milgrom Y 2001 Metabolism of gonadotropins: comparisons of the primary structures of the human pituitary and urinary $\mathrm{LH} \beta$ cores and chimpanzee CG $\beta$ core demonstrate universality of core production. Journal of Endocrinology 171 131-141.

Brockhausen I 1999 Pathways of O-glycan biosynthesis in cancer cells. Biochimica et Biophysica Acta 1473 67-95.

Elliott MM, Kardana A, Lustbader JW \& Cole LA 1997 Carbohydrate and peptide structure of the alpha and beta subunits of chorionic gonadotropin (hCG): characteristics and variants in 32 subunit preparations from normal and aberrant pregnancy and choriocarcinoma. Endocrine 7 15-32.

Ellish NJ, Saboda K, O’Connor J, Nasca PC, Stanek EJ \& Boyle C 1996 A prospective study of early pregnancy loss. Human Reproduction 11 406-412.

Fein HG, Rosen SW \& Weintraub BD 1980 Increased glycosylation of serum human chorionic gonadotropin and subunits from eutopic and ectopic sources: comparison with placental and urinary forms. Journal of Clinical Endocrinology and Metabolism 50 1111-1120.

Kobata A \& Takeuchi M 1999 Structure, pathology and function of the $\mathrm{N}$-linked sugar chains of human chorionic gonadotropin. Biochimica et Biophysica Acta 1455 315-326.

Kovalevskaya G, Birken S, Kakuma T \& O'Connor JF 1999a Early pregnancy human chorionic gonadotropin (hCG) isoforms measured by an immunometric assay for choriocarcinoma-like hCG. Journal of Endocrinology 161 99-106.

Kovalevskaya G, Birken S, Kakuma T, Schlatterer J \& O'Connor JF $1999 b$ Evaluation of nicked human chorionic gonadotropin content in clinical specimens by a specific immunometric assay. Clinical Chemistry 45 68-77.

Krichevsky A, Armstrong EG, Schlatterer J, Birken S, O’Connor J, Bikel K, Silverberg S, Lustbader JW \& Canfield RE 1988 Preparation and characterization of antibodies to the urinary fragment of the human chorionic gonadotropin beta-subunit. Endocrinology 123 584-593.

Krichevsky A, Birken S, O'Connor J, Acevedo HF, Bikel K, Lustbader J, Hartree A \& Canfield RE 1994a Development, characterization, and application of monoclonal antibodies to the native and synthetic beta $\mathrm{COOH}$-terminal portion of human chorionic gonadotropin (hCG) that distinguish between the native and desialylated forms of hCG. Endocrinology 134 1139-1145.
Krichevsky A, Birken S, O'Connor J, Bikel K, Schlatterer J \& Canfield R $1994 b$ The development of a panel of monoclonal antibodies to human luteinizing hormone and its application to immunological mapping and two-site assays. Endocrine 2 511-520.

Laemmli UK 1953 Cleavage of structural proteins during the assembly of the head of bacteriophage T4. Nature 227 680-685.

Laird NM \& Ware JH 1982 Random-effects models for longitudinal data. Biometrics 38 963-974.

Lapthorn AJ, Harris DC, Littlejohn A, Lustbader JW, Canfield RE, Machin KJ, Morgan FJ \& Isaacs NW 1994 Crystal structure of human chorionic gonadotropin (see comments). Nature 369 455-461.

Montgomery DC \& Peck EA 1982 Introduction to Linear Regression Analysis. New York: Wiley.

Nemansky M, Thotakura NR, Lyons CD, Reinhold BB, Reinhold VN \& Blithe DL 1998 Developmental changes in the glycosylation of glycoprotein hormone free alpha subunit during pregnancy. Journal of Biological Chemistry 15 12068-12076.

O'Connor JF, Schlatterer JP, Birken S, Krichevsky A, Armstrong EG, McMahon D \& Canfield RE 1988 Development of highly sensitive immunoassays to measure human chorionic gonadotropin, its beta-subunit, and beta core fragment in the urine: application to malignancies. Cancer Research 48 1361-1366.

O'Connor JF, Ellish N, Kakuma T, Schlatterer J \& Kovalevskaya G 1998 Differential urinary gonadotropin profiles in early pregnancy and early pregnancy loss. Prenatal Diagnosis 18 1232-1240.

Puett D \& Birken S 1989 Helix formation in reduced, S-carboxymethylated human choriogonadotropin beta subunit and tryptic peptides. Journal of Protein Chemistry 8 779-794.

Rosa C, Amr S, Birken S, Wehmann R \& Nisula B 1984 Effect of desialylation of human chorionic gonadotropin on its metabolic clearance rate in humans. Journal of Clinical Endocrinology and Metabolism 59 1215-1219.

Sauer MV, Paulson RJ, Francis MM, Macaso TM \& Lobo RA 1995 Preimplantation adoption: establishing pregnancy using donated oocytes and spermatozoa. Human Reproduction 10 1419-1422.

Skarulis MC, Wehmann RE, Nisula BC \& Blithe DL 1992 Glycosylation changes in human chorionic gonadotropin and free alpha subunit as gestation progresses. Journal of Clinical Endocrinology and Metabolism 75 91-96.

Taussky HH 1954 A microcolorimetric determination of creatinine in urine by the Jaffe reaction. Journal of Biological Chemistry 208 853-861.

Wide L \& Hobson B 1987 Some qualitative differences of hCG in serum from early and late pregnancies and trophoblastic diseases. Acta Endocrinologica 116 465-472.

Wu H, Lustbader JW, Liu Y, Canfield RE \& Hendrickson WA 1994 Structure of human chorionic gonadotropin at $2.6 \AA$ resolution from MAD analysis of the selenomethionyl protein. Structure 2 545-558.

Received in final form 22 October 2001 Accepted 30 October 2001 